\title{
Accumulation of Adrenaline in Sympathetic Nerve Endings in Various Organs of the Rat Exposed to Swimming Stress
}

\author{
Ayako SUDO \\ National Institute of Industrial Health, 21-1 Nagao 6-chome. \\ Tama-ku. Kawasaki 214. Japan \\ Accepted April 24, 1985
}

\begin{abstract}
Swimming produced a marked increase in adrenaline content, but produced no change or slight decrease in noradrenaline content in the heart. spleen and submaxillary gland of the rat. Pretreatment with desmethylimipramine abolished the swimming-induced increase in adrenaline in the three organs. It was also found that the administration of 6-hydroxydopamine caused almost complete depletion of both adrenaline and noradrenaline from these organs of control and swimmingtreated rats. Furthermore, a differential centrifugation study revealed that the microsomal fraction had the highest concentrations of both adrenaline and noradrenaline in the heart and spleen of control and stress-treated rats. These findings suggest that adrenaline. like noradrenaline, exists in sympathetic nerve endings in the peripheral organs of the rats under basal conditions, and the adrenaline contents in the nerve endings are increased under stress situations.
\end{abstract}

Adrenaline is a biologically active monoamine and has a great influence on various physiological functions. Small amounts of adrenaline are known to be contained in several organs of mammals in the normal condition (1-4). Hökfelt (1) reported that swimming stress increased adrenaline contents of the heart and liver of the rat. This finding suggests that adrenaline may be involved in stress reactions of these organs. In the view point of stress physiology, Hökfelt's finding should be confirmed, and physiological roles of adrenaline in the peripheral organs must be studied more precisely. However, few studies have been done for this purpose.

It has been demonstrated that stress causes an increase in circulating adrenaline level, which is due to enhanced release of adrenaline from the adrenal medulla (4). According to pharmacological studies $(5,6)$. exogenously administered adrenaline is taken up to various organs of mammals. Therefore, the increase in adrenaline content of the heart and liver during swimming stress may be attributable to increased uptake of adrenaline from the circulation. The previous studies $(5,6)$ also revealed that labeled adrenaline. like noradrenaline, is taken up to both neuronal and extraneuronal sites in the organs. It is expected that examination of where adrenaline increased during stress would provide some useful information about physiological roles of adrenaline in the organs.

Recent progress in high-performance liquid chromatography (HPLC) has made it possible to analyze minute amounts of adrenaline in the presence of noradrenaline. The present study was designed to elucidate the site of adrenaline accumulation in rat peripheral organs during swimming, using an improved method for the determination of catecholamines.

\section{Materials and Methods}

Animals: Male Sprague-Dawley rats (Clea Japan) were kept on 12-hr light-dark cycles. Food and water were given ad libitum. The ambient temperature and humidity were controlled at $25 \pm 1{ }^{\circ} \mathrm{C}$ and $55 \pm 5 \%$, respectively.

Sampling of blood and organs: At the times predetermined for killing the animals. they were anaesthetized with urethane and 
chloralose $(500 \mathrm{mg} / \mathrm{kg}$ and $50 \mathrm{mg} / \mathrm{kg}$. i.p., respectively) unless otherwise noted. Following a ventral abdominal incision, $3 \mathrm{ml}$ of arterial blood was drawn from the abdominal aorta into a syringe moistened with EGTA solution $(0.2 \mathrm{M}, \mathrm{pH} 6-7)$. Then various organs were quickly removed, rinsed with saline and blotted. Brains were dissected on ice according to the method of Glowinski and Iversen (7). Blood was centrifuged at $10^{4} \times \mathrm{g}$ for $15 \mathrm{~min}$, and reduced glutathione $(0.2 \mathrm{M}$. $60 \mathrm{al})$ as an antioxidant was added to the plasma. Centrifugation was carried out at $4^{\circ} \mathrm{C}$ in this study. The organs and plasma were stored at $-70^{\circ} \mathrm{C}$ until analysis.

Stress treatment: The rats were placed individually in polyethylene buckets $(50 \mathrm{~cm}$ i.d. and $60 \mathrm{~cm}$ in height) containing $50 \mathrm{~cm}$ of water, for 3.5 or $4 \mathrm{hr}$. The temperature of the water was maintained between $25^{\circ} \mathrm{C}$ and $28^{\circ} \mathrm{C}$. The experiment was carried out in the dark periods to minimize sleep deprivation in the rats during swimming. Blood and organs were obtained as mentioned above, immediately after the end of swimming. In the study on chemically sympathectomized rats described below, organs were removed $2 \mathrm{hr}$ after swimming.

Desmethylimipramine administration: Desmethylimipramine hydrochloride (Pertfran. Nippon Ciba-Geigy, $20 \mathrm{mg} / \mathrm{kg}$ ) dissolved in saline was administered to 6 -week-old rats. as a series of 3 i.p. injections 24,5 and $1 \mathrm{hr}$ before swimming exposure (8). The desmethylimipramine- and saline-treated rats were subjected to $3.5-\mathrm{hr}$ swimming, and blood and organs were taken as mentioned above.

6-Hydroxydopamine (6-OHDA) administration: Chemical sympathectomy was performed by a single administration of 6 OHDA (hydrobromide, Sigma, $68 \mathrm{mg} / \mathrm{kg}$ as free base). The drug was dissolved in saline containing ascorbic acid $(1 \mathrm{mg} / \mathrm{ml})$ and injected to the right external jugular vein of conscious rats. It was reported that noradrenaline in rat heart and spleen disappeared mostly $2 \mathrm{hr}$ after the administration of 6 OHDA (9). To examine the effect of chemical sympathectomy on the adrenaline and noradrenaline contents of the organs in control and stressed rats, 6-week-old animals were treated with 6-OHDA immediately after the end of 4-hr swimming, and 2 hr later, organs were removed under sodium pentobarbital anaesthesia ( $40 \mathrm{mg} / \mathrm{kg}$, i.p.)

Subcellular fractionation of organ homogenates: Each of the hearts and spleens obtained from control and stress-treated rats under sodium pentobarbital anaesthesia (40 $\mathrm{mg} / \mathrm{kg}$. i.p.) was homogenized in $10 \mathrm{ml}$ of $0.25 \mathrm{M}$ sucrose by using a glass homogenizer with a Teflon pestle in an ice bath. The sucrose homogenate was centrifuged at $10^{4} \times \mathrm{g}$ for $10 \mathrm{~min}$ and the supernatant was recentrifuged at $10^{5} \times \mathrm{g}$ for $60 \mathrm{~min}$ to obtain a microsome fraction, according to the method of Potter and Axelrod (10). The microsome fraction, the supernatant and the original sucrose homogenate were assayed for adrenaline and noradrenaline as described below. Smail portions of these samples were used for protein analysis by the method of Lowry et al. (11).

Measurement of adrenaline and noradrenaline in biological materials: The organs were weighed and homogenized in 10 volumes of ice-cold $0.4 \mathrm{M} \mathrm{HClO}_{4}$ solution with a Polytron homogenizer. The samples of differential centrifugation were treated in the same way. Plasma was mixed with a half volume of $1.5 \mathrm{M} \mathrm{HClO}_{4}$. The $\mathrm{HClO}_{4}$-treated samples were centrifuged, and adrenaline and noradrenaline in the supernatant were analyzed by a modification of the HPLCfluorometric method reported previously (12). Separation of the two catecholamines in alumina eluate of the organ extract was achieved by eluting a HPLC column of cation-exchange gel (TSK-IEX-510SP, $4 \times 300 \mathrm{~mm}$, Toyosoda) with $0.15 \mathrm{M}$ $\mathrm{NaH}_{2} \mathrm{PO}_{4}$ containing $10 \%$ acetonitrile. In the analysis of plasma catecholamines, a Nucleosil-5SA column (Macherey-Nagel, Chemcopak, $4 \times 200 \mathrm{~mm}$ ) and $0.15 \mathrm{M}$ $\mathrm{NaH}_{2} \mathrm{PO}_{4}$ containing $10 \%$ methanol were used.

Statistical analysis: Catecholamine contents of organs are expressed as nmol or pmol per g wet tissue, unless otherwise stated, and plasma levels are expressed as $\mathrm{pmol} / \mathrm{ml}$. The results represent the mean of the values from $6-10$ rats and S.E.M. The significance of the data was evaluated with 
the two-tailed Student's $t$-test.

\section{Results}

Effects of swimming on the concentrations of adrenaline and noradrenaline in plasma and various organs of the rats: Table 1 shows the concentrations of adrenaline and noradrenaline in plasma and various organs of the control and swimmingtreated rats. In control rats, the adrenaline contents in the heart, spleen and submaxillary gland were greater than the plasma level of adrenaline. Four-hour swimming in $27^{\circ} \mathrm{C}$ water produced marked increases in the adrenaline contents of these organs, while no change or slight decrease in noradrenaline level was observed. As a result, adrenaline to noradrenaline ratios in these organs were markedly increased after the stress. For instance, an 8-fold increase was found in the heart. Swimming-induced increase in adrenaline was also observed in other peripheral organs, but not in the brain regions. Adrenaline and noradrenaline in the adrenal gland were decreased by the swimming and plasma levels of adrenaline and noradrenaline were markedly elevated after the stress.

Effects of desmethylimipramine pretreat- ment on stress-induced increase in adrenaline contents of the rat organs: Table 2 indicates adrenaline and noradrenaline contents of the submaxillary gland, heart, spleen. adrenal gland and plasma of rats exposed and those unexposed to $3.5-\mathrm{hr}$ swimming after the administration of desmethylimipramine or saline. Following the swimming, the desmethylimipramine-treated rats did not show any significant increase in adrenaline in the submaxillary gland, heart and spleen, although the increase was significant in the salinetreated animals $(P<0.01)$. Plasma levels of adrenaline and noradrenaline in both the saline- and desmethylimipramine-treated groups were increased after the swimming. From the comparison of the saline-treated control group with the desmethylimipraminetreated control group in Table 2, it is apparent that the administration of desmethylimipramine caused a decrease in adrenaline and a slight increase in noradrenaline in the submaxillary gland, heart and spleen of the rats unexposed to the stress. These findings suggest that the peripheral organs accumulate considerable amounts of adrenaline by a neuronal uptake mechanism both in the basal state and in the stress situation.

Table 1. Changes of adrenaline and noradrenaline contents in rat organs after swimming stress

\begin{tabular}{|c|c|c|c|c|c|}
\hline & \multicolumn{2}{|c|}{ Noradrenaline } & \multicolumn{3}{|c|}{ Adrenaline } \\
\hline & Control & Swimming & Contro & & Swimming \\
\hline & \multicolumn{2}{|c|}{ (nmol/g) } & \multicolumn{3}{|c|}{$(\mathrm{pmol} / \mathrm{g})$} \\
\hline Heart & $4.42 \pm 0.27$ & $2.80 \pm 0.19^{* *}$ & $54.7 \pm 7.2$ & $\left(1.2^{\mathrm{a}}\right)$ & $284 \pm 22\left(9.2^{a}\right)^{* *}$ \\
\hline Spleen & $6.76 \pm 0.62$ & $4.25 \pm 0.42^{*}$ & $34.4 \pm 3.5$ & $(0.6)$ & $85.7 \pm 7.1(2.0)^{* *}$ \\
\hline Submaxillary gland & $8.02 \pm 0.37$ & $8.50 \pm 0.54$ & $8.2 \pm 1.5$ & $(0.10)$ & $135 \pm 24(1.6)^{* *}$ \\
\hline Lung & $0.60 \pm 0.04$ & $0.42 \pm 0.02^{* *}$ & $4.6 \pm 1.0$ & $(0.8)$ & $28.3 \pm 1.1(6.3)^{* *}$ \\
\hline Liver & $0.37 \pm 0.03$ & $0.37 \pm 0.03$ & $1.5 \pm 0.1$ & $(0.4)$ & $12.4 \pm 1.0(3.2)^{* *}$ \\
\hline Kidney & $1.01 \pm 0.11$ & $0.96 \pm 0.09$ & $12.1 \pm 2.9$ & $(1.2)$ & $69.0 \pm 6.0(6.7)^{* *}$ \\
\hline Abdominal wall muscle & $0.30 \pm 0.02$ & $0.28 \pm 0.01$ & $2.6 \pm 0.5$ & $(0.9)$ & $21.4 \pm 1.8(7.0)^{* *}$ \\
\hline Thigh muscle & $0.42 \pm 0.01$ & $0.33 \pm 0.01^{* *}$ & $2.2=0.3$ & $(0.5)$ & $21.4 \pm 1.5(6.0)^{* *}$ \\
\hline Vas deferens & $70.8 \pm 8.3$ & $73.5 \pm 7.0$ & $23 \pm 3$ & $(0.03)$ & $74 \pm 8 \quad(0.10)^{* *}$ \\
\hline Brain Hypothalamus & $7.85 \pm 0.37$ & $6.43 \pm 0.64^{*}$ & $162 \pm 15$ & $(2.0)$ & $(1.4)^{* *}$ \\
\hline Midbrain & $2.69 \pm 0.04$ & $2.42 \pm 0.19^{*}$ & $15.4 \pm 1.1$ & $(0.6)$ & $7.9 \pm 0.6(0.3)^{* *}$ \\
\hline Pons-meduila & $2.83 \pm 0.07$ & $2.63 \pm 0.16$ & $22.9 \pm 1.3$ & $(0.8)$ & $14.8 \pm 1.4(0.6)^{* *}$ \\
\hline Plasma (pmol/ml) & $1.6 \pm 0.1$ & $8.8 \pm 1.2^{* *}$ & $1.9 \pm 0.4$ & & $25.0 \pm 5.3$ \\
\hline Adrenal gland (nmol) & $38.6 \pm 1.9$ & $29.2 \pm 2.0^{* *}$ & $167 \pm 5$ & $(81.3)$ & $84 \pm 6 \quad(74.2)^{* *}$ \\
\hline
\end{tabular}

12-week-old male Sprague-Dawley rats were placed in $27^{\circ} \mathrm{C}$ water for $4 \mathrm{hr}$. Immediately after the swimming, blood was collected and organs were removed. Data represent the mean $\pm S . E . M .(N=6)$. $"$ Percentage of adrenaline. Ad/(Ad+NA) (\%) *: $P<0.05$ and **: $P<0.01$. compared to the control group. 
Effects of 6-OHDA administration on adrenaline and noradrenaline contents in the organs of the control and swimming-treated rats: Table 3 shows adrenaline and noradrenaline contents of the heart, spleen. submaxillary gland and adrenal gland of rats administered with 6-OHDA or vehicle immediately after 4-hr swimming or a sedentary period of the same duration and killed $2 \mathrm{hr}$ later. Swimming-induced increase in the

Table 2. Effects of desmethylimipramine pretreatment on swimming-induced increase in adrenaline content of the rat organs

\begin{tabular}{|c|c|c|c|c|}
\hline & \multicolumn{2}{|c|}{ Saline-treated } & \multicolumn{2}{|c|}{ DMI-treated } \\
\hline & Control & Swimming & Control & Swimming \\
\hline \multicolumn{5}{|l|}{ Noradrenaline } \\
\hline Heart $(\mathrm{nmol} / \mathrm{g})$ & $4.14 \pm 0.62$ & $2.57 \pm 0.23^{* *}$ & $4.89 \pm 0.21 * a$ & $3.92 \pm 0.56^{* * 1}$ \\
\hline Spleen $(\mathrm{nmol} / \mathrm{g})$ & $4.05 \pm 0.62$ & $3.96 \pm 0.81$ & $4.30 \pm 0.21$ & $3.17 \pm 0.39^{*}$ \\
\hline Submaxillary gland (nmol/g) & $7.59 \pm 0.67$ & $7.17 \pm 0.23$ & $8.49 \pm 0.78$ & $8.46 \pm 0.58$ \\
\hline Adrenal gland (nmol/pair) & $29.5 \pm 1.6$ & $23.9 \pm 1.5^{*}$ & $31.5 \pm 2.4$ & $24.5 \pm 1.8^{*}$ \\
\hline Plasma $(p m o l / m l)$ & $1.2 \pm 0.2$ & $7.9 \pm 0.8^{* *}$ & $3.3 \pm 0.2^{* *}$ & $20.5 \pm 4.6^{* *}$ \\
\hline \multicolumn{5}{|l|}{ Adrenaline } \\
\hline Heart (pmol/g) & $37.8 \pm 10.5$ & $187.4 \pm 19.9^{* *}$ & $15.9 \pm 1.4^{*}$ & $19.5 \pm 2.1$ \\
\hline Spleen $(p m o l / g)$ & $15.9 \pm 2.3$ & $81.8 \pm 10.8^{* *}$ & $8.1 \pm 0.7^{* *}$ & $8.6 \pm 1.2$ \\
\hline Submaxillary gland (pmol/g) & $11.0=2.1$ & $133.3 \pm 18.8^{* *}$ & $1.5 \pm 0.4^{* *}$ & $3.0 \pm 0.7$ \\
\hline Adrenal gland (nmol/pair) & $73.7 \pm 2.4$ & $29.8 \pm 3.2^{* *}$ & $65.3 \pm 2.8$ & $55.7 \pm 3.2^{*}$ \\
\hline Plasma $(p \mathrm{~mol} / \mathrm{ml})$ & $1.5 \pm 0.3$ & $14.4 \pm 3.3^{*}$ & $2.8 \pm 0.3$ & $6.4 \pm 2.0^{*}$ \\
\hline
\end{tabular}

Desmethylimipramine (DMl, hydrochloride, $20 \mathrm{mg} / \mathrm{kg}$, i.p.) or saline was administered to 6 -week-old rats, according to the injection schedule of Porsolt et al. (8), before 3.5-hr swimming (Swimming) or a sedentary period of the same duration (Control). Immediately after the swimming, blood and organs were obtained. Results represent the meantS.E.M. $(N=6-10)$. a: compared to the saline-treated control grcup (*: P<0.05. **:P<0.01). b: compared to the DMI-treated control group (*:P<0.05, $*$ * $P<0.01)$.

Table 3. 6-OHDA-induced decrease in adrenaline and noradrenaline contents of the organs of the rats exposed to swimming stress

\begin{tabular}{|c|c|c|c|c|c|c|c|}
\hline & \multicolumn{3}{|c|}{ Contral } & \multicolumn{4}{|c|}{ Swimming } \\
\hline & & Vehicle & 6-OHDA & $\begin{array}{c}\text { 6-OHDA/ } \\
\text { Vehicle } \\
(\%)\end{array}$ & Vehicle & 6.OHDA & $\begin{array}{c}\text { 6-OHDAI } \\
\text { Vehicle } \\
(\%)\end{array}$ \\
\hline \multirow[t]{2}{*}{ Heart } & NA & $4.86 \pm 0.30^{a}$ & $0.11 \pm 0.01$ & $\left(2.2^{b}\right)$ & $3.73 \pm 0.14^{* * c}$ & $0.18 \pm 0.02$ & $(4.8)$ \\
\hline & Ad & $68.2 \pm 7.3$ & $7.2 \pm 1.4$ & $(10.5)$ & $149 \pm 24^{* *}$ & $6.0 \pm 1.0$ & $(4.0)$ \\
\hline \multirow[t]{2}{*}{ Spleen } & NA & $3.96 \pm 0.25$ & $0.18 \pm 0.04$ & $(4.6)$ & $4.41 \pm 0.38$ & $0.66 \pm 0.04$ & $(14.9)$ \\
\hline & Ad & $21.5 \pm 1.8$ & $2.2 \pm 0.6$ & $(10.5)$ & $60.4 \pm 7.8^{* *}$ & $7.6 \pm 1.1$ & $(12.6)$ \\
\hline \multirow{2}{*}{$\begin{array}{l}\text { Submaxillary } \\
\text { gland }\end{array}$} & $N A$ & $8.03 \pm 0.85$ & $0.08 \pm 0.01$ & $(1.0)$ & $7.41 \pm 0.39$ & $0.17 \pm 0.01$ & $(2.3)$ \\
\hline & $\mathrm{Ad}$ & $24.6 \pm 4.4$ & $0.74 \pm 0.31$ & $(3.0)$ & $116 \pm 13^{* *}$ & $1.01 \pm 0.14$ & $(0.9)$ \\
\hline \multirow{2}{*}{$\begin{array}{l}\text { Adrenal gland } \\
\text { (nmol/pair) }\end{array}$} & NA & $19.8 \pm 0.9$ & $21.5 \pm 1.0$ & $(108.5)$ & $18.9 \pm 1.1$ & $15.4 \pm 1.4^{* d}$ & $(81.0)$ \\
\hline & $\mathrm{Ad}$ & $61.8 \pm 1.9$ & $70.5 \pm 3.4$ & $(114.1)$ & $24.7 \pm 1.4^{* *}$ & $25.1 \pm 2.6^{* *}$ & (101.5) \\
\hline
\end{tabular}

6-OHDA (68 mg/kg. i.v.) or vehicle was administered to 6-week-old rats immediately after 4-hr swimming. and 2 hr later, the organs were removed. ": The values are the mean \pm S.E.M. $(N=6)$ expressed as $\mathrm{nmol} / \mathrm{g}$ wet tissue for noradrenaline (NA) and as $\mathrm{pmol} / \mathrm{g}$ wet tissue for adrenaline (Ad), unless otherwise stated. b: Data in parentheses are the amine value of 6-OHDA-treated animals divided by the value of the vehicletreated ones (\%). " : **, P<0.01. compared to the control group treated with vehicle. "t: ${ }^{*}, P<0.05$ and ${ }^{*}, P<0.01$, compared to the control group treated with 6-OHDA. 
adrenaline level in the heart, spleen and submaxillary gland of the vehicle-treated rats was still observed $2 \mathrm{hr}$ after the cessation of swimming exercise. Intravenous administration of 6-OHDA led to almost complete depletion of adrenaline, as well as noradrenaline, in the three organs. This was recognized in both of the control and stresstreated groups. The degree of 6-OHDAinduced decrease in adrenaline was almost equal to that of noradrenaline, especially in the stress-treated group. These findings suggest that both adrenaline and noradrenaline are contained in sympathetic nerve endings in these three organs of rats under normal and stress situations.

Subcellular localization of adrenaline and noradrenaline in rat heart and spleen: Table 4 gives the subcellular distributions of adrenaline and noradrenaline in the hearts and spleens of control and swimmingtreated rats studied by differential centrifugation. Catecholamine contents are expressed as pmol per $\mathrm{mg}$ of protein. The noradrenaline-rich fraction. which was the sediment obtained after the centrifugation at $10^{5} \times \mathrm{g}$ for $60 \mathrm{~min}$, contained a larger amount of adrenaline than the amounts in the supernatant and the original homogenate. The adrenaline to noradrenaline ratio was virtually the same among the catecholamine- rich fraction, the supernatant and the original homogenate in each of the control and swimming-treated groups. It should be noted that swimming produced a significant increase in adrenaline content of the noradrenalinerich fraction of the heart $(P<0.05)$. A similar tendency was observed in the microsomal fraction of the spleen. It is probable, therefore, that the swimming stress increased the adrenaline content of the noradrenalinestoring particles in the two organs of rats.

\section{Discussion}

Several workers have reported that small but measurable amounts of adrenaline are contained in rat plasma, heart, submaxillary gland, spleen and some other organs (1-4. 13-15). The present study confirmed the presence of adrenaline in the rat organs, although the values are smaller than most of the values in the previous studies. From the present results, it is obvious that the amounts of adrenaline in the heart, spleen and submaxillary gland are higher than the plasma level of adrenaline in control rats. Therefore, it is unlikely that adrenaline extractable from these three organs is derived from the blood and extracellular fluid contained in the respective organs. The heart, spleen, submaxillary gland and vas deferens contain greater amounts of noradrenaline compared

Table 4. Subcellular localization of adrenaline and noradrenaline in the heart and spleen of the rats exposed to swimming stress

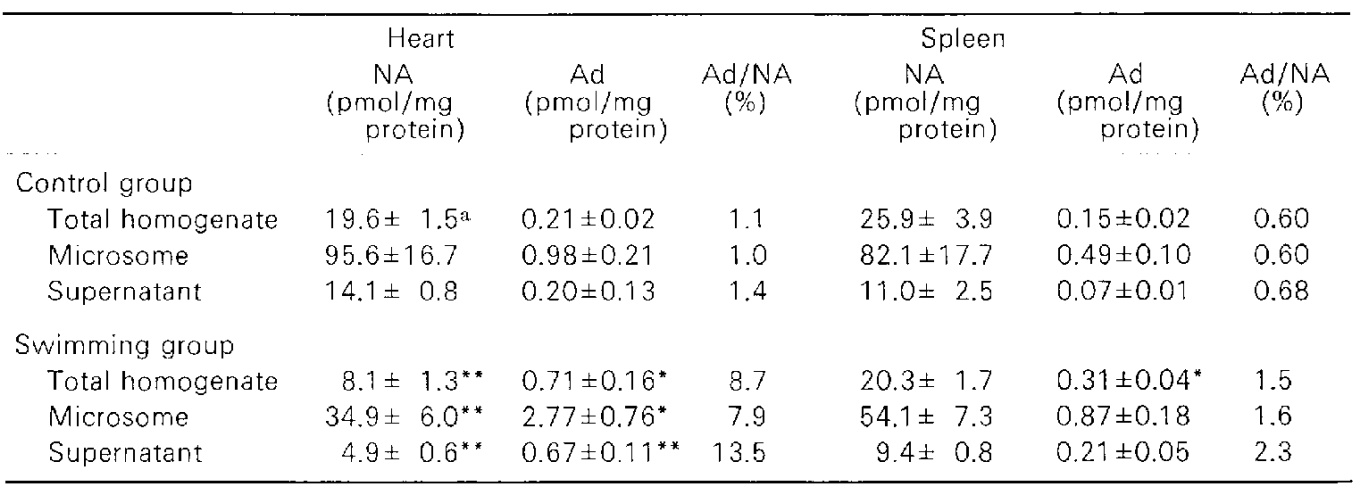

12-week-old rats were treated with 4-hr swimming. The hearts and spleens of control and swimmingtreated rats were homogenized in $0.25 \mathrm{M}$ sucrose and centrifuged at different speeds. Microsome means the fraction sedimenting between $10^{4} \mathrm{~g} \times 15 \mathrm{~min}$ and $10^{5} \mathrm{~g} \times 60 \mathrm{~min}$ centrifugation of the sucrose homogenate. "V: Values are the mean $=$ S. E.M. $(N=6)$. NA: Noradrenaline. Ad: Adrenaline. *: $P<0.05$ and **: $P<0.01$, compared to the control group. 
with the amounts in other extra-adrenal peripheral organs, and those noradrenalinerich organs are also rich in adrenaline.

The present work confirmed that swimming induces an increase in adrenaline and no change or slight decrease in noradrenaline in the rat heart and liver as described by Hökfelt (1), and it further indicated that these changes occur in other extra-adrenal peripheral organs of rats. It is known that noradrenaline turnover in various organs is accelerated during exposure to stress such as foot-shock, cold. restraint, treadmill or swimming $(16,17)$. The decrease in noradrenaline content of several organs during the swimming in the present study may be ascribed to enhanced release of noradrenaline accompanied by increased sympathetic nerve activities. The swimming increased plasma adrenaline and noradrenaline levels and decreased adrenal contents of adrenaline and noradrenaline. Since plasma adrenaline, even in a stress situation. mostly disappears after adrenal medullectomy (4), the increase in plasma adrenaline during the swimming may be attributable to enhanced release of the amine from the adrenal medulla.

The adrenaline increase in the peripheral organs by the swimming stress could be due to enhanced synthesis of adrenaline. increased uptake of adrenaline, or reduction in its utilization. Although little is known about the adrenaline turnover rate in the organs during stress, it was reported that swimming in $34^{\circ} \mathrm{C}$ water caused an enhancement of the turnover rate of noradrenaline in the rat heart (17), as described above. On the other hand, it is known that phenylethanolamine- $\mathrm{N}$-methyl transferase (PNMT) activities in rat heart and spleen are considerably lower than the activities in the adrenal gland and brain stem. whereas the adrenaline content of the heart is higher than the content of the brain stem (3). Stress-induced increase in PNMT activity in the adrenal gland was observed after repeated stress exposure, but not after acute stress (18). These observations suggest that the increase in the adrenaline content of the peripheral organs after the stress exposure is not due to the increase in the rate of synthesis. In the present study. swimming-induced increase in adrenaline was observed only in peripheral organs, but not in the brain. This is probably because most of the circulating adrenaline is unable to penetrate the blood-brain barrier (5). In view of these facts. it is likely that the increase of adrenaline in the organs during stress resulted from increased uptake of adrenaline to the organs.

It has been demonstrated that exogenously administered adrenaline and noradrenaline are taken up to various organs in mammals ( 5 . 6 ). The previous studies have revealed that the catecholamine uptake process consists of two different mechanisms, neuronal and extraneuronal uptake. According to the study of Lightman and Iversen (19), the rate of the neuronal uptake of exogenous adrenaline in the isolated rat heart is nearly equal to the rate of extraneuronal uptake at perfusion concentrations below $0.05 \mathrm{nmol} / \mathrm{ml}$. Adrenaline and noradrenaline incorporated into neuronal sites are retained for at least several hours, whereas catecholamines in extraneuronal sites are metabolized quickly by COMT and MAO (6). Therefore, no substantial accumulation of catecholamine in extraneuronal regions could be observed with such a low concentration of catecholamine in the perfusion medium. At higher perfusion concentrations, the rate of extraneuronal uptake of catecholamine exceeds the metabolic capacity of the degradative enzymes, so that extraneuronal accumulation of catecholamines occurs (6).

Adrenaline level in rat plasma was less than $3 \mathrm{pmol} / \mathrm{ml}$ under the basal condition and not higher than $30 \mathrm{pmol} / \mathrm{ml}$ immediately after swimming in the present experiment. It is assumed, therefore, that adrenaline is present largely in neuronal sites in the peripheral organs of the animal and that the swimming-induced increase in adrenaline is probably attributable to neuronal uptake rather than extraneuronal uptake. This assumption was tested, in the present study, by pharmacological and biochemical experiments on several adrenaline- and noradrenaline-rich organs. The study demonstrates that pretreatment with desmethylimipramine, a neuronal uptake blocker, abolished the swimming stress-induced adrenaline increase in rat heart, spleen and submaxillary gland, 
suggesting neuronal uptake of adrenaline to the organs during the stress. In the rats unexposed to swimming stress, adrenaline contents of these three organs were reduced by the administration of desmethylimipramine. It appears that neuronal uptake of adrenaline to the organs occurs even under basal conditions. Intraneuronal existence of adrenaline in resting and stress conditions is supported by the present result of chemical sympathectomy. That is, the administration of 6-OHDA caused almost complete depletion of both adrenaline and noradrenaline from the heart, submaxillary gland and spleen in control and stress-treated rats. This finding strongly suggests the presence of adrenaline in noradrenergic nerve endings in the organs of the rat.

Biochemical studies have demonstrated that most of the noradrenaline contained in sympathetically innervated organs is stored in particles which are observed by electron microscopy as dense-cored vesicles (20). Potter (21) showed that noradrenalinestoring particles in rat cardiac tissue can be purified by differential centrifugation and that the microsomal fraction obtained was enriched 7 -fold over the original homogenate in terms of noradrenaline per mg protein. The microsomal fraction prepared from whole rat heart in the present work had 5-fold higher concentration of noradrenaline, and the noradrenaline-rich fraction contained greater amounts of adrenaline than those in the supernatant and the original homogenate. Interestingly, the adrenaline content in the noradrenaline-rich fraction was greatly increased after the swimming stress. A similar result was obtained from the spleen.

Taken together, it is concluded that adrenaline exists in sympathetic nerve endings, probably in noradrenaline-storing particles, in rat organs such as the heart. spleen and submaxillary gland, and it is indicated that the accumulation of adrenaline in the organs during swimming stress is attributable to neuronal uptake of circulating adrenaline.

Rosell et al. (22) demonstrated that ${ }^{3} \mathrm{H}$ adrenaline taken up from the circulation to isolated dog gracilis muscle was released following sympathetic nerve stimulation.
Several workers have proposed the adrenaline-neurotransmitter hypothesis based on the results of physiological experiments $(23,24)$. On the other hand, it has been reported that more than one neurotransmitter coexists in certain neurons (25). One possible explanation for the physiological significance of neurons with multiple messengers is that different neuronal messages are transferred with different ratios of the transmitter components (25). The present work suggests that sympathetic neurotransmitter in mammals is a mixture of at least two components, noradrenaline and adrenaline. It is also demonstrated that the ratio of adrenaline to noradrenaline is increased during and follow ing stress exposure. It is expected, therefore, that an increased amount of adrenaline will be released by the nerve impulse, resulting in augumented responses of $\beta$-adrenoceptors in sympathetic neurotransmission. This process might be involved in stress reactions of the sympathetic nervous system.

Acknowledgements: The author is grateful to Prof. M. Otsuka. Tokyo Medical and Dental University, for his kind advice. She is also indebted to Dr. S. Yamamoto, National Institute of Industrial Health, for his interest in this work. Desmethylimipramine was kindly supplied by Nippon Ciba-Geigy.

\section{References}

1 Hökfelt, B.: Noracrenaline and adrenaline in mammalian tissues. Acta Physiol. Scand. 25, Supp. 92, (1951)

2 Anton, A.H. and Sayre, D.F.: A study of the factors affecting the aluminum oxide-trihydroxyindole procedure for the analysis of catecholamines. J. Pharmacol. Exp. Ther. 138, 360-375 (1962)

3 Pendleton, R.G., Gessner, G. and Sawyer, J.: Studies on the distribution of phenylethanolamine $\mathrm{N}$-methyltransferase and epinephrine in the rat. Res. Commun. Chem. Pathol. Pharmacol. 21. 315-325 (1978)

4 Kevetnansky, R., Weise, V.K., Thoa, N.B. and Kopin, I.L.: Effects of chronic guanethidine treatment and adrenal medullectomy on plasma levels of catecholamines and corticosterone in forcibly immobilized rats. J. Pharmacol. Exp. Ther. 209, 287-291 (1979)

5 Axelrod, J., Weil-Malherbe, H. and Tomchick, R.: The physiological disposition of $\mathrm{H}^{3}-$ epinephrine and its metabolite metanephrine. J. 
Pharmacol. Exp. Ther. 127, 251-256 (1959)

6 Iversen, L.L.: The Uptake and Storage of Noradrenaline in Sympathetic Nerves. Cambridge University Press, Cambridge (1967)

7 Glowinski, J. and Iversen, L.L.: Regional studies of catecholamines in the rat brain. I. The disposition of ${ }^{3} \mathrm{H}$-norepinephrine. ${ }^{3} \mathrm{H}$-dopamine and ${ }^{3} \mathrm{H}$-dopa in various regions of the brain. $J$. Neurochem. 13, 655-669 (1966)

8 Porsolt, R.D., Anton, G., Blavet, N. and Jalfre, $M$.: Behavioral despair in rats; a new model sensitive to antidepressant treatments. Eur. J. Pharmacol. 47, 379-391 (1978)

9 Thoenen, $H$. and Tranzer, J.P.: Chemical sympathectomy by selective destruction of adrenergic nerve endings with 6-hydroxydopamine. Naunyn Schmiedebergs Arch. Pharmakol. Exp. Pathol. 261, 271-288 (1968)

10 Potter, L.T. and Axelrod, J.: Properties of norepinephrine storage particles of the rat heart. J. Pharmacol. 142, 299-305 (1963)

11 Lowry, O.H., Rosebrough, N.J., Farr, A.L. and Randall, R.J.: Protein measurement with the Folin phenol reagent. J. Biol. Chem. 193, 265$275(1951)$

12 Sudo, A.: Measurement of adrenaline in the rat brain by high-performance liquid chromatography with fluorometric detection. Ind. Health 20, 151-156 (1982)

13 Popper, C.W., Chiueh, C.C, and Kopin, I.J.: Plasma catecholamine concentrations in un. anesthetized rats during sleep. wakefulness immobilization and after decapitation. J. Pharmacol. Exp. Ther. 202, 144-148 (1977)

14 Strömblad, B.C.R. and Nickerson, M.: Accumulation of epinephrine and norepinephrine by some rat tissues. J. Pharmacol. Exp. Ther. 134, 154-159 (1961)

15 von Euler, U.S, and Ryd, G.: Effect of sympathetic denervation and adrenalectomy on the catecholamine content of the rat submaxillary gland. Acta Physiol. Scand. 59, 62-66 (1963)

16 Stone, E.A.: Stress and catecholamines. In Catecholamines and Behaviour. Edited by Friedhoff. A.J., Vol. 1, p. 31-72, Plenum Press. New York and London (1975)

17 Östman-Smith, 1., Sjöstrand, N.O. and Swedin, G.: Cardiac noradrenaline tumover and urinary catecholamine excretion in trained and untrained rats during rest and exercise. Acta Physiol. Scand. 86, 299-308 (1972)

18 Kvetnansky, R., Weise, V.K. and Kopin, I.J.: Elevation of adrenal tyrosine hydroxlase and phenylethanolamine- $\mathrm{N}$-methyl transferase by repeated immobilization of rats. Endocrinology 87, 744-749 (1970)

19 Lightman, S.L. and Iversen, L.L.: The role of uptake 2 in the extraneuronal metabolism of catecholamines in the isolated rat heart. Br. J. Pharmacol. 37, 638-649 (1969)

20 Smith, A.D.: Subcellular localization of noradrenaline in sympathetic neurons. Pharmacol. Rev. 24, 435-457 (1972)

21 Potter, L.T.: Role of intraneuronal vesicles in the synthesis, storage and release of noradrenaline. Circ. Res. 21. Supp. 3, 13-24 (1967)

22 Rosell, S., Axelrod, J. and Kopin, I.J.: Release of tritiated epinephrine following sympathetic nerve stimulation. Nature 201, 301 (1964)

23 Brown, M.J. and MacQuin, 1.: Catecholamine neurotransmitters and the heart. Acta Med. Scand. Supp. 660, 34-39 (1982)

24 Berecek, K.H. and Brody, M.J.: Evidence for a neurotransmitter role for epinephrine derived from the adrenal medulla. Am. J. Physiol. 242, H593-H601 (1982)

25 Hökfelt, T., Johansson, O., Ljungdahl, A., Lundberg, J.M. and Schultberg, M.: Peptidergic neurons. Nature 284, 515-521 (1980) 